\title{
MS44-P05 | EVALUATION OF CRYSTAL STRUCTURES WITH PARTIAL OCCUPANCIES USING \\ BOOLEAN SATISFIABILITY TECHNIQUES IN STRUPLOX
}

Fischer, Reinhard (Universität Bremen, Bremen, GER); Soeken, Mathias (EPFL, Lausanne, CH); Drechsler, Rolf (Universität Bremen, Bremen, GER)

The crystal-chemical evaluation of crystal structures is usually based on the interpretation of interatomic distances and angles, as, e.g., done just recently [1] evaluating more than 7000 crystal structures of zeolites in terms of the values of their bondlengths $\mathrm{T}-\mathrm{O}(\mathrm{T}=\mathrm{Si}, \mathrm{Al}, \mathrm{P}, \mathrm{Zn}, \mathrm{Be}, \mathrm{Ge}, \mathrm{B}, \mathrm{As}, \mathrm{Ga}, \mathrm{Co})$ and their variability. This approach works well for ordered structures with well-defined distances. If atom positions are partially occupied, the interpretation of crystal structures becomes difficult or nearly impossible if there are multiple positions with partial occupancies. In order to find a solution for possible atom distributions where the simultaneous occupancy does not yield conflicting distances to nearest neighbors, we have employed Boolean satisfiability (SAT) techniques [2] assigning Boolean variables to each atom position in the unit cell being true if it is occupied and false if it is vacant. Restrictions due to site occupancies calculated in crystal-streucture refinements are encoded as Boolean expressions which are then combined and passed to the SAT algorithm. Here we present the program STRUPLOX providing an automated procedure for the interpretation of the crystal structure starting with the input of atom parameters until the presentation of possible solutions for the atom distribution. Restrictions for interatomic distances are derived from the sum of ionic radii of adjacent atoms. Interatomic distances are calculated and coordination numbers are determined from bond-valence sum calculations.

[1] Baur, Fischer. Chem. Mater. 31 (2019) 2401-2420

[2] Soeken, Drechsler, Fischer. Z. Kristallogr. 231 (2016) 107-111 